Disclosure of Interests: Thurston Yan Jia Heng: None declared, Nicholas Chew: None declared, Kexin Amanda Choo: None declared, Aisha Lateef: None declared, Manjari Lahiri Grant/research support from: Manjari Lahiri is the site principal investigator for the Singapore National Biologics Register, which is a multi-pharmaceutical funded register, in which industry sponsors provide support through the Chapter of Rheumatologists, Singapore. Dr Lahiri does not personally receive any remuneration.

DOI: 10.1136/annrheumdis-2020-eular.761

\section{THU0261 NEW 2019 SLE EULAR/ACR CLASSIFICATION CRITERIA ARE VALID FOR IDENTIFYING SLE AMONG PATIENTS ADMITTED FOR PERICARDIAL EFFUSION}

L. Delaval ${ }^{1}$, T. Goulenok ${ }^{1}$, A. Dossier ${ }^{1}$, T. Papo ${ }^{1}$, K. Sacre ${ }^{1} .{ }^{1}$ Université Paris Diderot, Paris, France

Background: The new 2019 SLE European League Against Rheumatism/American College of Rheumatology (EULAR/ACR) classification criteria for systemic lupus erythematosus (SLE) have been recently published. Seritis is a prominent -often inaugural- feature of active SLE. Low titers of antinuclear antibodies (ANA) have been frequently reported in patients with idiopathic pericarditis. Of note, ANA positivity at a titer $\geq 1 / 80$ is now mandatory as an entry criterion in the 2019 SLE EULAR/ACR classification criteria.

Objectives: Although classification criteria have theoretically no individual diagnostic purpose, we aimed at testing this new criteria set in unselected patients with pericardial effusion

Methods: In a retrospective study performed in the Department of Internal Medicine, University Paris Diderot, a French competence centre for rare systemic autoimmune diseases (AID), all consecutive adult patients hospitalized from January 2009 to January 2019 for pericardial effusion were reviewed. Clinical and biological data collected at time of the diagnosis of pericardial effusion were analyzed. The characteristics of the patients are listed in Table 1. Three sets of lupus criteria (SLE ACR-1997, SLE SLICC and 2019 SLE EULAR/ACR criteria) were applied in all ANA-positive patients

Results: Over a 10-year period, 137 patients were admitted for pericardial effusion. Search for ANA was systematically performed at diagnosis in all but $8(n=129)$ and measured at a titer $\geq 1: 80$ on Hep- 2 cells in 49 patients $(38 \%)$ that were eventually separated in three groups: $17(34.7 \%)$ patients with a final diagnosis of SLE based on senior clinician judgement, $6(12.2 \%)$ patients with a final diagnosis of autoimmune disease (AID) other than SLE (primary Sjögren's syndrome $(n=2)$, undifferentiated connective-tissue disease $(n=2)$ and systemic sclerosis $(n=2))$ and $26(53.1 \%)$ patients with a diagnosis of idiopathic pericarditis after exclusion of malignancy, tuberculosis and systemic inflammatory diseases with a median 12.3 [1.6-29.8] months follow-up

The 2019 SLE EULAR/ ACR criteria were met in 100\% of patients with SLE, $33.3 \%$ of patients with non-SLE AID and $11.5 \%$ of patients with idiopathic pericarditis. Thus this new set of criteria for SLE offered a higher sensitivity $(100 \%)$ but a lower specificity (84.38\%) as compared to the former criteria, for the diagnosis of SLE in patients with pericardial effusion. Interestingly, the 2019 SLE EULAR/ACR classification score was higher in SLE patients (median: 30 [11-45]) as compared to non-SLE AID (median: 8 [6-12], $\mathrm{p}=0.0006$ ) and idiopathic pericarditis patients (median: 6 [5-12], $\mathrm{p}<0.00001$ ). Moreover, the 2019 classification set score strongly correlated with the SLEDAI activity score [6] as shown Figure $S 1\left(R^{2}\right.$ $=0.8105, p<0.00001)$. Setting the 2019 SLE EULAR/ACR classification threshold score $>12$ (out of a theoretical maximum of 51 ) instead of $\geq 10$ increased the specificity of 2019 SLE EULAR/ ACR criteria from $84.38 \%$ to $100 \%$. Overall, in patients with pericardial effusion and positive ANA, the diagnosis of SLE could be ruled out when 2019 SLE EULAR/ACR criteria score was < 10 and confirmed when the score was $>12$.

Conclusion: This study shows that the new 2019 SLE EULAR/ACR criteria for SLE are helpful in clinical practice for the diagnosis of SLE in patients admitted for pericardial effusion

Acknowledgments: Jean-François Alexandra, Marie Berleur, Marie-Paule Chauveheid, Gregory Ducrocq, Damien van Gysel, Diane Rouzaud Disclosure of Interests: None declared

DOI: 10.1136/annrheumdis-2020-eular.348

\section{THU0262 LEVELS OF OSTEOCALCIN AND PROCOLLAGEN TYPE I C-TERMINAL PROPEPTIDE IN PATIENTS WITH SYSTEMIC LUPUS ERYTHEMATOSUS, THEIR ASSOCIATION WITH BONE MINERAL DENSITY AND LEVEL OF INTERLEUKIN-6}

S. Shevchuk ${ }^{1}$, L. Denyshchych ${ }^{2} .{ }^{1}$ National Pirogov Memorial Medical University, Scientific and Research Institute of Invalid Rehabilitation (Educational Scientific Treatment Complex) of National Pirogov Memorial Medical University, Vinnytsia, Ukraine; ${ }^{2}$ National Pirogov Memorial Medical University, Vinnytsia, Ukraine
Background: Osteoporosis and fractures associated with it are considered to be one of the most severe complications of systemic lupus erythematosus (SLE). The role of a systemic inflammatory process, vitamin D deficiency, hypogonadism and peculiarities of disease treatment in reduced bone mineral density (BMD) is being discussed. Even though the frequency of osteoporosis in patients with SLE is being studied extensively by scientists from different countries, data on the peculiarities of bone tissue metabolism and the factors that provoke disorders of bone remodeling in such individuals are quite limited. The association between markers of bone tissue metabolism and BMD, and how they change during an inflammatory process is poorly studied.

Objectives: The objective of our research is to study the levels of osteocalcin $(\mathrm{OC})$ and procollagen type I C-terminal propeptide (PICP) in patients with systemic lupus erythematosus and to estimate their association with BMD and inflammatory activity based on the levels of interleukin-6 (IL-6).

Methods: A total of 58 women with SLE (the average age was $45.11 \pm 1.03$ years old) and 29 individuals from the control group (the average age was 46.79 \pm 2.30 years old) were examined. The diagnosis of SLE was established on the basis of 2019 EULAR/ACR classification criteria for SLE. Levels of IL-6, OC and $\mathrm{PICP}$ in serum were determined by enzyme immunoassay. Changes in BMD of the lumbar spine at the level of L1-L4 and the proximal femur were determined by dual-energy X-ray absorptiometry. In postmenopausal women, the diagnosis of osteoporosis was established by the T-score $\leq-2.5$ SD. Osteopenia met T-score from -1 to -2.5 SD. In women of reproductive age, the Z-score was used to determine BMD. Values of the Z-score $\leq-2.0 \mathrm{SD}$ were considered as "below expected range for age".

Results: The average OC level in serum of practically healthy individuals equaled $17.64 \pm 0,59 \mathrm{ng} / \mathrm{ml}$, and in patients with SLE it was $13.96 \pm 0.40 \mathrm{ng} / \mathrm{ml}$, i.e. it was $20.9 \%$ lower. The average PICP level in the control group equaled $107.8 \pm 4.28 \mathrm{ng} / \mathrm{ml}$, in the main group it was $92.9 \pm 5.01 \mathrm{ng} / \mathrm{ml}$, i.e. $16 \%$ lower Overall, the decrease in the bone turnover markers (PICP and/or OC) was noticed in 28 patients with SLE $(48.3 \%)$ and only in 4 practically healthy individuals $(13.8 \%)$.

In women with decreased bone turnover markers, the T-score of the lumbar spine and hip was 2.3-2.6 times lower $(p<0.05)$ than in the group with adequate levels of bone turnover markers. Z-score was also lower among patients with decreased levels of OC and PICP. In this group, the average BMD leve was $0.81 \pm 0.05 \mathrm{~g} / \mathrm{cm}^{2}$ and was $13.8 \%$ lower than in the group of patients with no signs of bone tissue metabolism disorder $-0.94 \pm 0.02 \mathrm{~g} / \mathrm{cm}^{2}$. Among the group of women with signs of suppression of biosynthetic processes in bone tissue, there were twice more individuals with decreased BMD. In patients with critically high levels of IL-6 (above $20.0 \mathrm{ng} / \mathrm{L}$ ), OC level was lower than in patients with high (12.5-20.0 ng/L) and adequate $(<12.5 \mathrm{ng} / \mathrm{L})$ levels of IL- 6 (by 17.3 and $19 \%$ respectively). The proportion of individuals with low OC levels increased from $31.2 \%$ in the last group to $70.6 \%$ among patients with critically high levels of IL-6.

PICP level was also lower (38.1\% and $39.7 \%$ respectively) in case of critically high IL-6 levels compared to its high and adequate levels. The proportion of individuals with low PICP levels increased from $6.3 \%$ in the group with adequate IL-6 level to $58.8 \%$ in the group with critically high IL-6 level.

Conclusion: Women with SLE have bone tissue metabolism disorder in the form of decreased bone turnover markers (procollagen type I C-terminal propeptide and osteocalcin) associated with the inflammatory activity. In the group of patients with the signs of suppression of biosynthetic processes in the bone tissue, there were more individuals with decreased BMD.

Disclosure of Interests: Sergii Shevchuk Grant/research support from: Celltrion, Inc, Liudmyla Denyshchych: None declared DOI: 10.1136/annrheumdis-2020-eular.2604

\section{THU0263 \\ LUNG INVOLVEMENT IN PRIMARY SJÖGREN SYNDROME - AN UNDER-DIAGNOSED ENTITY}

G. Sogkas ${ }^{1}$, S. Hirsch ${ }^{1}$, K. Olsson ${ }^{2}$, R. Schmidt ${ }^{1}$, T. Witte ${ }^{1}$, A. Jabonka ${ }^{1}$, D. Ernst ${ }^{1} .{ }^{1}$ Medical Highschool Hannover, Clinic for Immunology and Rheumatology, Hannover, Germany; ${ }^{2}$ Medical Highschool Hannover, Clinic for Pneumology, Hannover, Germany

Background: Interstitial lung disease (ILD) represents a frequent extra-glandular manifestation of primary Sjögren's Syndrome (pSS). Limited published data regarding phenotyping and treatment exists. Advances in managing specific ILD phenotypes have not been comprehensively explored in patients with coexisting pSS.

Objectives: This retrospective study aimed to phenotype lung diseases occurring in a well-described pSS cohort and describe treatment course and outcomes. Methods: Between April 2018 and September 2019, all pSS patients attending our Outpatient clinic were screened for possible lung involvement. Clinical, laboratory and computer tomography (CT) findings were analysed. Patients were classified according to CT findings into 5 groups: usual interstitial pneumonia 